\title{
Access Control and Recording System Using SQLite and RFID
}

\author{
Yalcin Ezginci (Corresponding author) \\ Department of Electrical and Electronics, Faculty of Engineering and Natural Science, \\ Konya Technical University, 42075, Konya, Turkey \\ E-mail: yezginci@ktun.edu.tr \\ Meryem Karadas \\ Department of Electrical and Electronics, Faculty of Engineering and Natural Science, \\ Konya Technical University, 42075, Konya, Turkey
}

\begin{abstract}
In this study, a prototype system, of which recordings are made into the SQLite database, which is controlled by an Arduino microcontroller, which approves or rejects the accesses to be made through a gate by reading RFID tags with an RFID reader, was realized. Door access, control, and recording operations are provided with an interface designed with $\mathrm{C \#}$. The contact, date, time, and minute information of accesses is displayed and recorded with the SQLite database. The system consists of two parts. The prototype door section consists of an Arduino Uno microcontroller, RFID reader, LCD, DC motor, DC motor drive, and a sliding door. A computer, recording and control software created with C\#, and the SQLite database running in relation to this software are found in the second part.
\end{abstract}

Keywords: Access Control, RFID, Arduino, SQLite database

DOI: $10.7176 / \mathrm{JSTR} / 5-10-04$

\section{Introduction}

RFID, which means identification with radio frequency, refers to data transmission by radio waves. It is one of the automatic identification technologies used to obtain information about people, animals, or products standing in a place or moving. The data are stored in a transporter (tag) carrying it (Finkenzeller, 2010). RFID solutions can easily meet a large number of deficiencies in identifiers, such as a barcode. Storing more information in the RFID tag means more information about them that can be used. RFID also provides the monitoring and data collection opportunity in environments that are not suitable for employees. Without a direct line of sight between the RFID tag and the reader, but of course, at a certain distance and under certain conditions, more than a thousand readings per second can be performed with excellent accuracy. Data on an RFID tag can be changed over and over again (Thakare 2008). These provide advantages to the RFID system in many applications compared to a barcode and other identifiers. The fact that semiconductor chips for data storage can be realized at technological and affordable prices, they can be used easily, and they can be integrated into computer systems brings them to the forefront.

\subsection{RFID Historical Background}

The beginning of RFID was in October 1948 with the publishment of Harry Stockman's article "Communication by Means of Reflected Power." The system of identification of friend or foe (IFF), which is popular in the aircraft, was one of the first applications of RFID. During the rapid development of microelectronics technology in the 1970s, companies, universities, and government laboratories were actively involved in the development of practical RFID applications such as animal tracking, vehicle tracking, and factory automation. Since the 1990s, many technological advances such as microelectronics, embedded software, and wireless networks have expanded the functionality of RFID dramatically (Lehpamer, 2012). RFID technology makes it possible for manufacturers, retailers, and suppliers to manage inventory, business processes, and security audits easily, quickly, and efficiently. With different sectors and services, the visibility of RFID is increasing, and the solutions it offers are expanding. 


\subsection{RFID System Overview}

Unlike barcodes that a user scans by spending time by using hands and by observing, the information about the object or human stored in the microchip of the RFID tag is obtained in a shorter time, without contact and automatically. With the latest advances in technology, efforts, which are aimed at utilizing RFID in automatic identification and data capture processes and determining new applications increase. An RFID system consists of a tag to identify the object, a reader/writer, and a computer to control and record the received data or an interface such as USB, RS232, and RS 485, which can transfer the data to a robot control system as a sample (Thakare 2008). The data are built into the tag; a reader/writer reads the tag data, or the contents are changed. The data received from the reader can be recorded and evaluated in a database.

Typical carrier frequencies (transmission frequency of the reader) in today's applications are between $125 \mathrm{kHz}$ and $2.45 \mathrm{GHz}$. Possible application areas for non-contact identification systems have also recently increased. Many applications of RFID systems have become widespread worldwide. The technical parameters of systems such as urban transport ticket, animal identification, industrial automation, or access control have been optimized for a variety of application areas. When designing RFID systems, several requirements such as operating frequency, maximum range, safety requirements, memory capacity, standards and application (product type, product price, and company-specific) requirements must be taken into account (Thakare). The application to be performed can go up to a high level of complexity depending on the transfer of data, the encryption of communication, the inclusion of the set of protocols and the data processing capabilities to provide them.

In this study, a passive RFID tag was used. The most important feature of the passive tag is that it does not contain a power source or battery and therefore requires external power. The RFID system is based on the electromagnetic interaction of the coils (antennas) communicating between the tag and the reader. In this system, the reader transfers power to the tag via the magnetic field, and then data are transferred from the tag to the reader. The power and data transfer is realized through the air gap (through the opening) between the tag and the reader. This situation means a two-way communication structure. Therefore, the tag and the reader comprise a radio frequency module and a coil. The chip in the tag is activated only when an RFID reader scans it. The reader transfers some energy to the chip, and the activated chip transmits the data on it to the reader again through the electromagnetic path using a carrier signal (RF), after passing them through the coding and modulation processes. The reader obtains its unique identifier number on the tag after RF demodulation, filtering, and decoding stages.

\subsection{RFID Working Principles}

There is an electromagnetic (contactless) exchange between the tag carrying the data and the reader which will read them. This exchange has different operating modes according to the distance (opening) between the tag and the reader, depending on being close coupling $(0-1 \mathrm{~cm})$, remote coupling $(0-1 \mathrm{~m})$, and long-range $(>1 \mathrm{~m})$. The read/write distance of the RFID system used is designed for the maximum desired range. In this study, the design will be made according to the close coupling system. The communication procedure between the transmitter and the reader is carried out by the use of "reflection or backscatter." The mutual transmission frequencies are the same (1:1) (Thakare, 2008). Frequencies in these systems range from $100 \mathrm{kHz}$ to $30 \mathrm{MHz}$.

Close coupling is done according to the inductive coupling technique, which we are familiar with from circuit theory. The transfer of energy and data is possible by the mutual inductance (M) between the two coils. As in the transformer, the primary conductor creates a magnetic field around an electric current passing through the coil, and a voltage is induced in a secondary conductor coil exposed to this magnetic field. In the RFID system, a drive coil (reader) and a receiver coil (tag) for power transfer and impedance matching circuits related to them are designed. In this way, the magnetic field that will be generated by the reader coil at the resonant frequency is increased, on the other hand, the dimensions of the tag coil are reduced, while the magnetic flux density in the tag is tried to be made maximum in order to increase the transferred power. The inductive connection between the two coils functions as a communication channel. According to equation (1.1), the proportion of the energy captured by the secondary coil can be represented by the coupling coefficient, $\mathrm{k}$ :

$$
\mathrm{k}=\mathrm{M} / \mathrm{L} 1 * \mathrm{~L} 2
$$

where $\mathrm{k}$ is the coefficient of coupling $(0 \leq \mathrm{k} \leq 1), \mathrm{L} 1$ and $\mathrm{L} 2$ are the inductance of the coils, M is the mutual inductance. The mutual inductance depends only on the geometry of the two coils. The coefficient 
of coupling, i.e. $\mathrm{k}$ value, is the indicator of how closely the two coils are linked magnetically and is an essential factor for power transmission. However, since the air gap in RFID systems is small (typical values for $\mathrm{k}$ in a few centimeters are 0.1 or 0.01 ), the two coils will have been fairly weakly coupled. $\mathrm{k}$ is obtained with the theoretical calculations made depending on the diameters of the coils and the distance between them. For an example, $\mathrm{k}=0.0213$ is obtained when the tag diameter is 0.4 inch, the reader diameter is $5 \mathrm{inch}$, and the distance between them is $1 \mathrm{inch}$. The voltage value transferred to the load in the secondary of the transformer can be obtained by equation (1.2).

$$
\mathrm{U}_{2}=\mathrm{B} * \mathrm{k} * \mathrm{i}
$$

Here, k belongs to the geometry of the coils and the air gap and may be within a specific value range. B is a linear scale factor or a coefficient representing the system gain. As the variable which can be controlled, the current which will flow from the primary coil remains. By changing the current value of the primary coil, the voltage on the tag coil can be increased to the desired levels (Lehpamer, 2012). Although there is a weak connection in RFID, a coil current (rms) value of $100 \mathrm{~mA}$ is sufficient in practice. At this stage, the inductive coupling becomes applicable as an interface, which is cost-effective, low-power, and high-bandwidth, for interconnecting modules on the chip, such as RFID.

\subsection{Similar studies in the literature}

In a study, it was determined that the Fast Passing System (FPS) was established at Dokuz Eylül University (Izmir-Turkey) campus entrances with intensive entrance and exit, processes were modeled, and performance measurements were made, and the speed of passing through the campus gates was doubled (Tecim, 2016).

Ez et al. (2018) established an RFID-based automatic access control system using Arduino. When the RFID reader installed in the system detects an RFID tag, the tag is read and is compared with IDs in the memory, and access is granted if the ID is a recorded one; otherwise, access is denied.

Cobantoro and Anugra (2017) designed a car parking application based on VB.Net and MySQL by using RFID technology for the parking system at their university. At the entrance, users are guided with the process of determining the parking space. The RFID parking system was observed to be successful in setting parking spaces and in finding the parking space shown to users.

Deniz et al. (2017) established an RFID lock system, which is controlled by Arduino, for detecting the institutional identity of the personnel in laboratories or private areas of educational institutions. Users in these areas can be detected externally.

Bektaş and Sondaş (2016) established a system by using RFID technology in order to control the entrance and exit of a laboratory. It was aimed to perform the security audit by recording the date and time when the authorized personnel enter and exit. In this context, an interface with Microsoft C \# was created, and for the identity information of the personnel, the Microsoft SQL 2008 database was used.

\section{Material and Method}

This section briefly describes the components used in the design of the transition system.

\subsection{Arduino Uno}

Arduino Uno is a microcontroller board based on ATmega328. It includes 14 digital input/output pins (6 of which can be used as PWM (pulse width modulation) outputs), six analog inputs, a $16 \mathrm{MHz}$ ceramic resonator, a USB, a reset button and everything needed to support a microcontroller. The Arduino IDE is the software development environment, and it is the proprietary software program of Arduino based on $\mathrm{C} / \mathrm{C}++$.

\subsection{RFID Card Reader}

FEATURES

Communication speed: $424 \mathrm{kbit} / \mathrm{s}$

Operating Voltage: $3.3 \mathrm{~V}$

Operating Frequency: $13.56 \mathrm{MHz}$

Operating Current: 13-26mA

Sleep Current: $80 \mathrm{uA}$

Communication Protocol: SPI

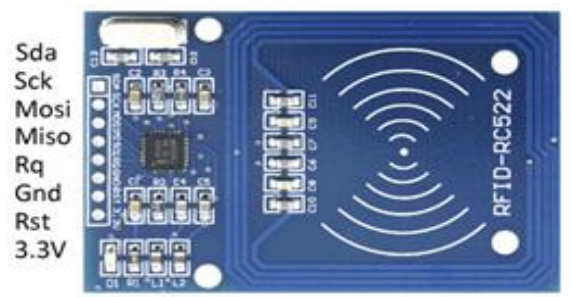

Figure 1. RC522 13.56 MHz RFID Reader Module and Technical Specifications 


\section{$2.3 I^{2} \mathrm{C}$ protocol}

Besides the requirement of using at least nine connection wires between the Arduino and LCD module, an intelligent solution to overcome other problems that may arise is to use the $\mathrm{I}^{2} \mathrm{C}$ protocol. Accordingly, between the two modules (ICs), which include a plurality of connections, an $\mathrm{I}^{2} \mathrm{C}$ module must be added to communicate over two lines. This module transfers data over Sda and Scl pins, except the feed pins. First of all, the $\mathrm{I}^{2} \mathrm{C}$ protocol library must be added to the Arduino. With the ability to initiate data transfer from a bus, a module takes over the role of a master and the transfer of data to the connected modules (slaves) is carried out.

\subsection{Serial Communication Protocol}

Serial communication means that the signals to be transmitted are sent consecutively over a single line. USB, RS232, and Ethernet ports perform serial communication with the computer. In order for the transmitted data to be delivered bug-free to the other party, the number of bits that the sending device and the receiving device can send in one second must be the same. This data transmission rate is called the baud rate. The baud rate is the number of bits carried in 1 second. For example, if the transmission rate is 9600 Baud, the processing time of one bit of information is 104 microseconds.

\subsection{Motor Control with Pulse Width Modulation (PWM)}

The speed of the DC motor is controlled by changing the input voltage. The most basic way to change the input voltage is to generate PWM signals. Pulse width modulation changes the average value of the voltage via the electronic doors which open and close quickly. The average voltage occurs as the ratio of the time the signal is "on" to the time the signal is "off" in a period. (As shown in Figure 2.a, a low voltage average is obtained at short pulse intervals, and a high voltage average is obtained at wide pulse intervals.)

\subsection{H-Bridge and L298N Integrated Circuit}

The direction of the current in the motor is reversed in order to change the direction of the DC motor rotation. However, if the motor will rotate in both directions, this should be prepared as hardware. Hbridge configuration provides this solution. In the H-bridge, four switching elements, such as thyristors or transistors, form a structure in a way that the motor is in the center and columns are H-like. According to the direction of the voltage, the rotation in both directions takes place with the completion of the circuit via the transverse half-arms in a way that the current is passed through the motor at each time. As observed in Figure 2.b, switches S1 and S4 are turned off to rotate the DC motor in the direction of the arrow, while switches S2 and S3 are open circuits. In order for the DC motor to rotate in the opposite direction, the switches must be brought to the reverse state of their previous position. Thus, when the PWM and H-Bridge structure are combined, full control over the DC motor will be provided.
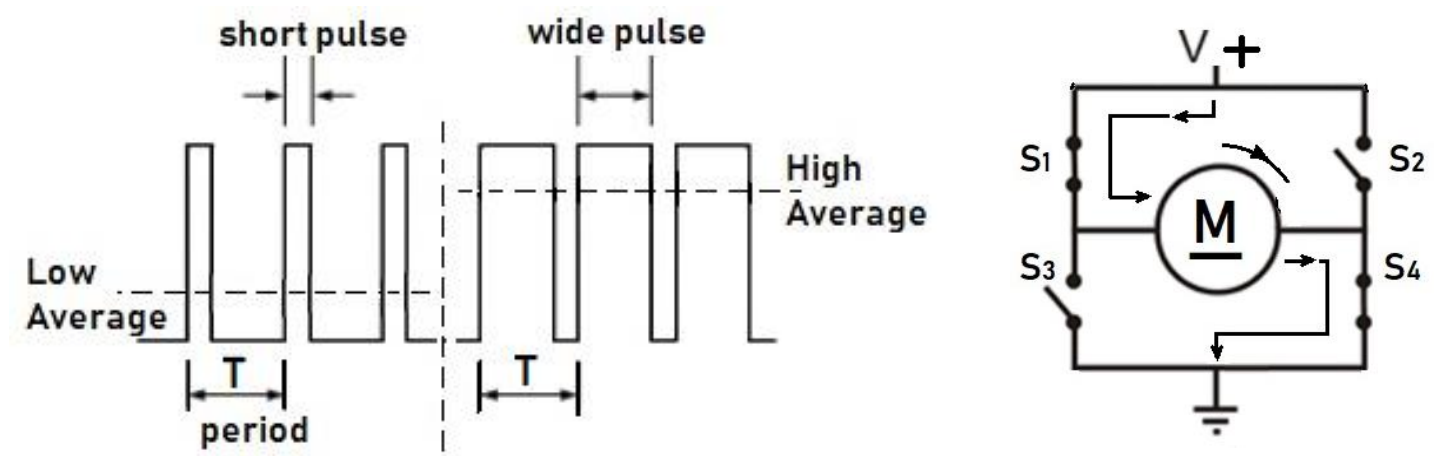

Figure 2.a Average voltage change with pulse width modulation

Figure 2.b DC motor direction change

The DC motor drive with these features is L298N from the integrated circuits. The DC motor drive module with LM298N was used here. (Figure 2) The module can operate DC motors that have a voltage between $5 \mathrm{~A}$ and $35 \mathrm{~V}$, with a peak current of up to $2 \mathrm{~A}$. 


\subsection{SQLite Database}

SQLite is a relational SQL database engine that is widely distributed in the world and has fully opensource codes. The SQLite database is encoded in $\mathrm{C}++$ and has a compact structure. It can be easily used with almost any programming language such as C\#, PHP, ASP, Delphi, and Perl. Since it does not need any server to run, there are no installation or configuration steps. There is only one file for each database. This situation makes it easier to back up and copy the database (Kreibich, 2010). Before programming with C\#, the reference to System. Data.SQLite must be added by marking the related .dll. At the same time, "using System.Data.SQLite;" should be added to the encoding.

\section{Design}

Security systems play an important role in preventing unauthorized persons from entering areas containing physical and intellectual property. Locked doors among basic safety devices may not provide adequate security. A prototype RFID accessing system was designed to monitor people's movements and restrict access to unauthorized areas. The principal scheme of the accessing system is presented in Figure 3. The RFID system consists of RFID tags (cards) to be identified, a reader mounted on the secure entrance, and an LCD controlled by the Arduino Uno microcontroller, and a prototype door accessing system. The door accessing system consists of a DC motor and driver, led warning, liquid crystal display (LCD), and a mechanical sliding door mechanism.

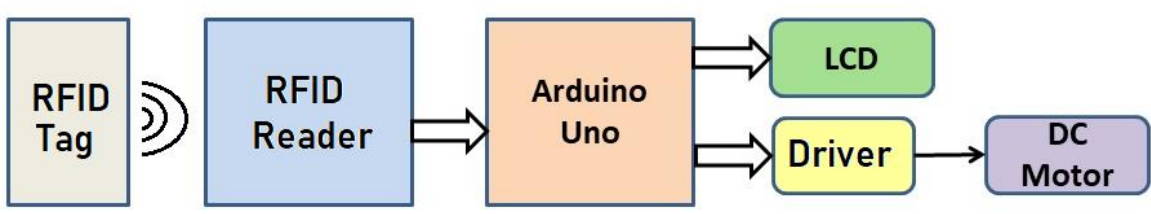

Figure 3. Principle Diagram of the System

The algorithm of the software of the accessing control system was shown in Figure 4. For this, firstly, the tag IDs to be allowed to access must be read by the reader and saved in the database.

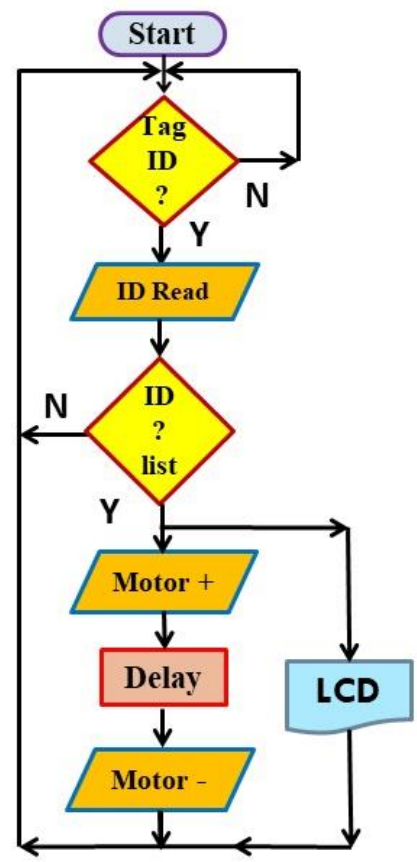

Figure 4. Flow Diagram 


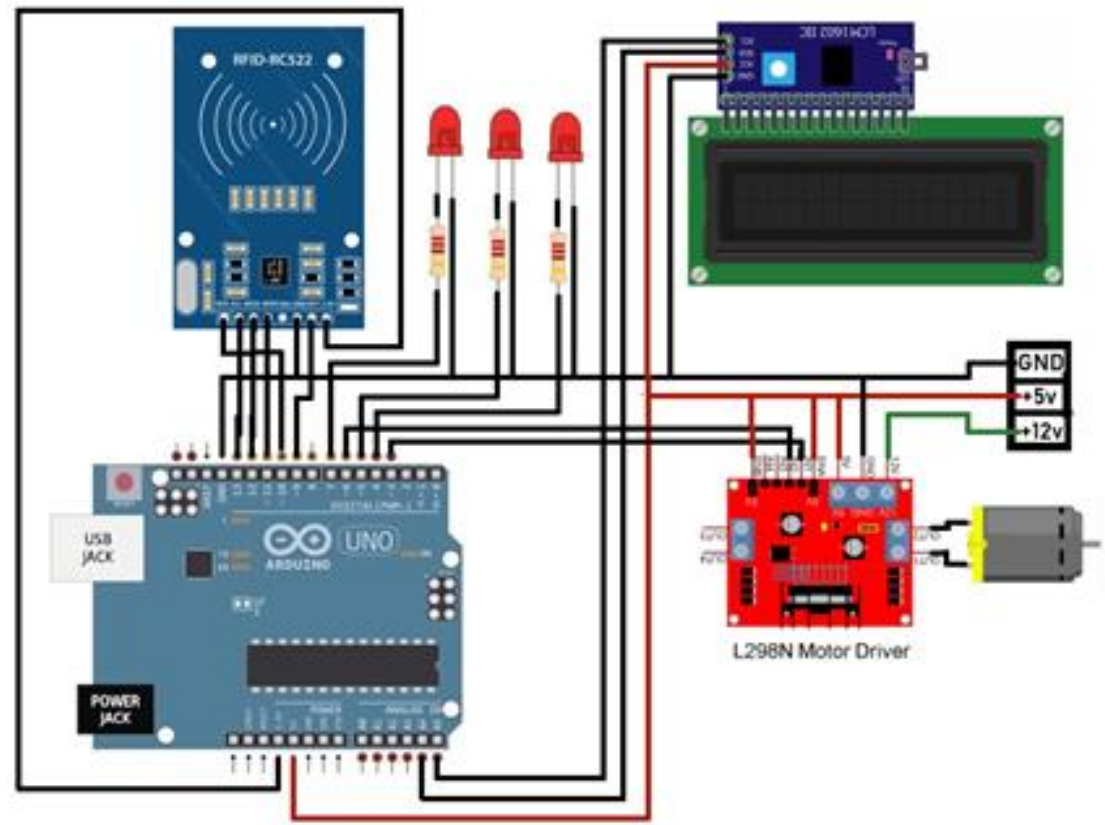

Figure 5. Circuit Diagram

Once the system is made functional, it is kept ready to read a label at any moment. When a tag is approximated and its ID is read, it is checked whether this identification exists in the database. If not, the program returns to the tag reading position. If the read ID is present in the database, it processes the date and time at that moment together with its information to the database, shows it on the user operations screen and indicates that it is confirmed by the LED light. At the same time, it runs the DC motor in the forward direction for a certain period, and the door is opened, and after waiting for 5 seconds, the door is closed by running in the reverse direction this time. The schematic diagram of the electronic circuits, including the connection of the hardware belonging to the designed door accessing system, was demonstrated in Figure 5. Figure 6 displays the prototype accessing system, which realizes the implementation of the processes of which operation is explained in the flow diagram.

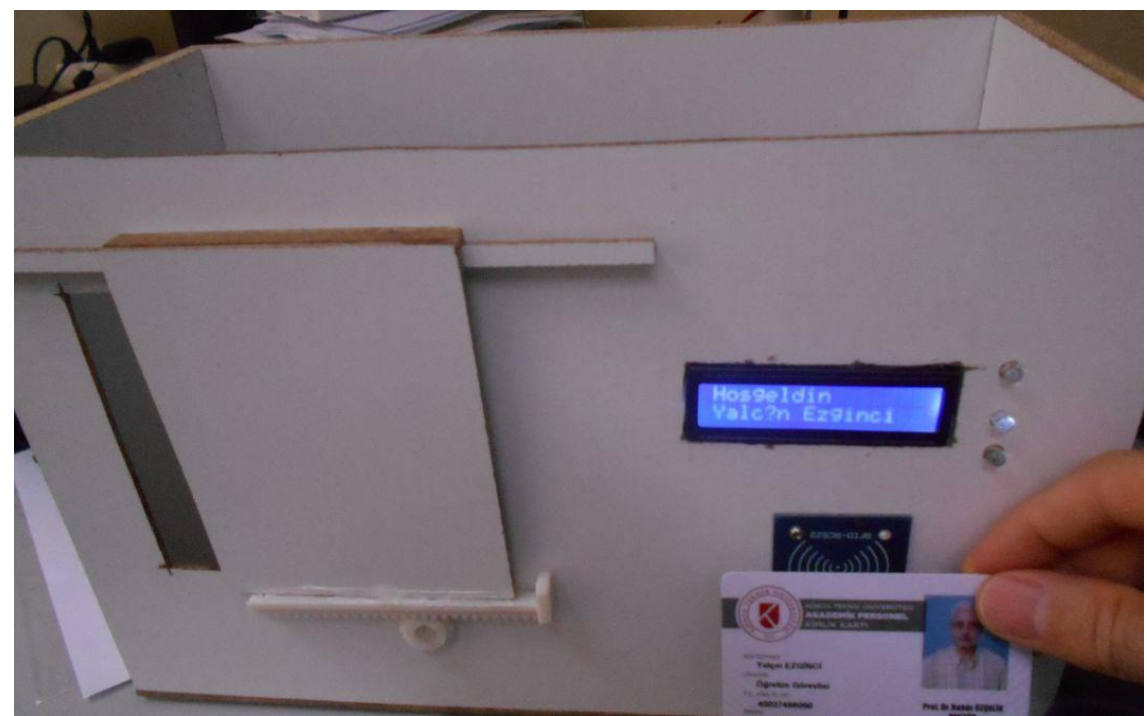

Figure 6. The prototype of the realized accessing System 
LEDs turn on their warning lights according to the authorized and unauthorized attempts. When the system is run, the control interface is displayed first, as shown in Figure 7.a.

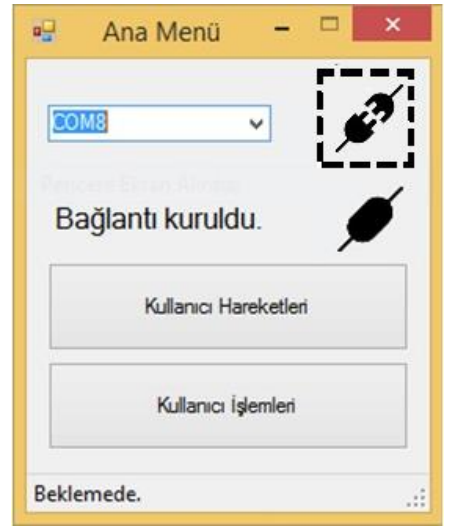

Figure 7.a Main menü

\begin{tabular}{|c|c|c|c|c|}
\hline \multicolumn{3}{|c|}{ 吗 Kullanıcı Hareketleri } & $\square$ & $\times$ \\
\hline Id & İsim & Soyisim & Tarih & $\wedge$ \\
\hline 194 & Şeyma & Erkul & 07.08.2019 10:53:43 & \\
\hline 195 & Hakan & Atalay & 07.08.2019 10:53:51 & \\
\hline 196 & Yalq̧n & Ezginci & $07.08 .201910: 54: 03$ & \\
\hline 197 & Hakan & Atalay & 07.08.2019 11:05:49 & \\
\hline 198 & Yalq̧n & Ezginci & 07.08.2019 11:07:26 & \\
\hline 199 & Yalan & Ezginci & 07.08.2019 11:07:40 & $\checkmark$ \\
\hline
\end{tabular}

Figure 7.b User movements screen

At the first moment, as it can be seen in the dashed square, the connection could not be established, and the "select port" warning appears in the ComboBox tool. The names of the computer's USB ports are listed here in COM3 or similar way. When the selection is made, and the connection button of the plugs is pressed, the connected form of the upper plugs is formed, and the "Connection established" message is printed in the label box. Afterward, by pressing the other two buttons, the Windows Form screenshots linked to the database are obtained.

Figure 7.b shows the user movements screen. This screen is again a table and lists the information about the records in progress in order and is used for monitoring the entries. In the far left, the number of entries, information about the one who enters, date and time can be observed.

While the DC motor rotates a gear, thin rectangular chipboard with a tooth structure was placed on it (Fig. 6). Thus, the circular motion of the motor is converted to linear planar motion. In this way, the back and forth movement of the rectangular plate on the gear represents the opening and closing of the entrance. As shown in Figure 8, the ID identifications of the user tags are made and recorded in the database system. When the RFID tag containing the unique information of the user is scanned by the RFID reader and matched with the information in the database, Arduino starts the drive and the DC motor by generating a confirmation code, and at the same time, displays the user name and card number on the LCD, and waits for the user to pass. After 5 seconds, the motor rotates in the opposite direction and closes the door automatically.

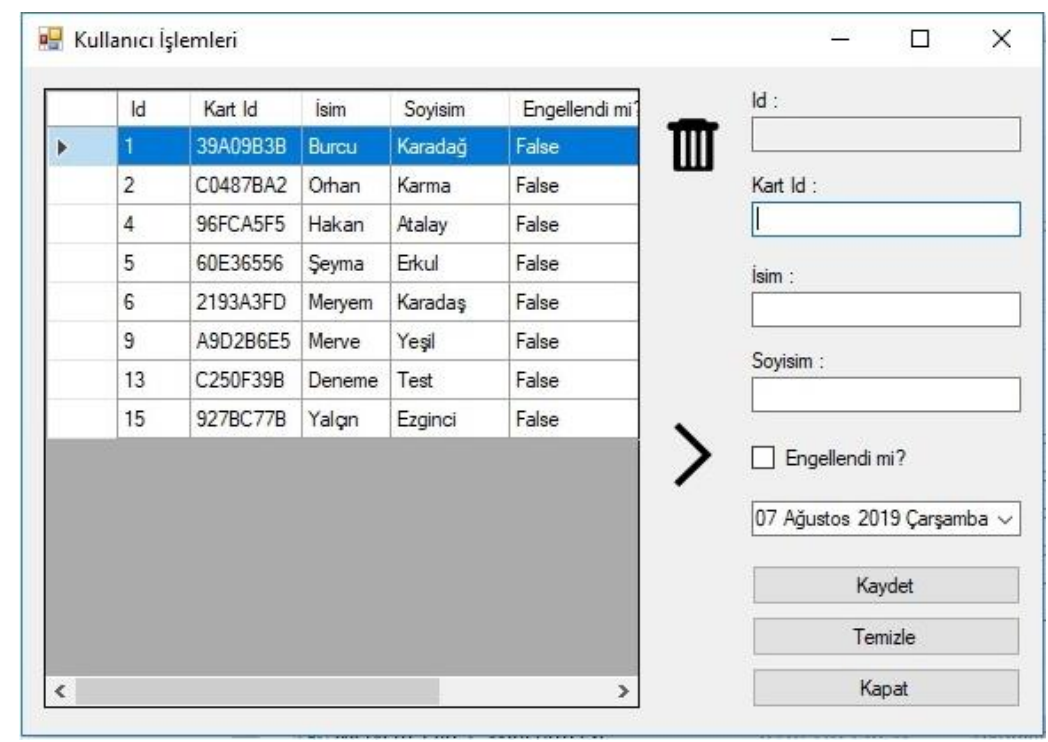

Figure 8. User identification procedures 
From the user identification operations screen shown in Figure 8, the ID of the user's tag is read, an index number, which is automatically given by the database and which shows in which order the user is registered, the code of the tag, User name, surname information, and blocking status (correct /incorrect) information are entered. The program completes the recording by automatically processing the date and time data that this recording process is included in the system. At any time, the access status of a person may be suspended. This situation is indicated by the right or wrong sign in the "is he/she blocked" column in that person's database. It is possible to access and update these recording operations at any time during the process.

The door accessing system provides access to authorized personnel by using RFID technology and denies access to unauthorized personnel. Users are given a tag and are recorded in the database system with ID identification, as shown in the figure. If authorization limitation is to be made, it will be again marked over this interface.

\section{Conclusion and Recommendations}

The access control and recording system with RFID, which has an SQLite database, was realized on a prototype door with a sliding door. Those with authorized RFID tags are allowed to enter the area where this system is installed, and if they are not authorized, no access is allowed. After the passing, the door is closed in a short period like 5-10 seconds. Furthermore, the tag ID of all attempts that are allowed and not allowed to access is recorded until its date, hour, and minute into the database. In this application, to use a public transportation card (Hand-Card) and a student and staff card of the university directly for access is a significant advantage. The infrastructure of this study is easy, inexpensive, extensible, and it has a structure that can be added to computer network systems. In the continuation of this study, this system may be established with the purpose of making secure and monitoring the entries and exits to the private areas of public and private enterprises with the additions deemed to be necessary. Furthermore, since student and staff ID cards in universities are often RFID-tagged, this application can be used at the entrances to classrooms or laboratories.

\section{References}

Bektaş F., Sondaş A., (2016), RFID Modülü İle Kapı Giriş-Çıkış Takip Sistemi Uygulaması, Düzce Üniversitesi Bilim ve Teknoloji Dergisi, 4 (2016) 603-608

Cobantoro AF., Anugra FG., (2017), Design of a parking system using RFID based on VB and MYSQL, VOLT JurnalImiah PendidikanTeknik Elektro Vol. 2, No. 2, October 2017, 109-116

Ez O., Oleka CV, Nduanya UI, (2018). Automatic Access Control System using Arduino and RFID, Journal of Scientific and Engineering Research, 5(4):333-340, ISSN: 2394-2630

Finkenzeller, K., (2010), RFID Handbook: Fundamentals and Applications in Contactless Smart Cards, Radio Frequency Identification and Near-Field Communication, Third Ed. John Wiley \& Sons, Ltd

Kreibich JA., (2010), Using SQLite, O’Reilly Media, Inc., USA, ISBN: 978-0-596-52118-9

Lehpamer, (2012), RFID Design Prenciples, Harvey Lehpamer, 2012, Artech House

Özdemir D., Ceylan O., Ulusoy A. (2017), RFID Kart Sistemi İle Personel Odas1, Sinıf ve Laboratuvar, Giriş Kontrolü, The Journal of Graduate School of Natural and Applied Sciences of Mehmet Akif Ersoy University Special Issue 1: 134-139.

Tecim V., Toplallar M., Emç M., Şentürk S., Aydın C., (2016), RFID Tabanlı Hızlı Geçiş Sistemleri İçin İş Akışlarının Yönetim Amaçlı Modellenmesi, Yönetim Bilişim Sistemleri Dergisi, Cilt:1, Say1:3, 2016, Sayfa 307-317

Thakare Y. B., Musale S.S., Ganorkar S.R., (2008) A Technological Review of RFID \& Applications, IET Conference on Wireless, Mobile and Multimedia Networks, 2008 p. $65-70$ 